\title{
31. GEOCHEMISTRY OF INTERSTITIAL WATERS
}

\subsection{INTERSTITIAL WATER STUDIES ON SMALL CORE SAMPLES FROM THE MEDITERRANEAN SEA ${ }^{1}$}

\author{
F. L. Sayles and L. S. Waterman, Woods Hole Oceanographic Institution, Woods Hole, Massachusetts \\ and \\ F. T. Manheim, U. S. Geological Survey, Woods Hole Oceanographic Institution, Woods Hole, Massachusetts
}

\begin{abstract}
Of ten Leg 13 sites studied by us, eight give definite evidence of the existence of halite-containing sediments beneath the seabed. This conclusion is based on the existence of continuous sodium and chloride enrichments in interstitial waters with depth. This is the only direct evidence of the existence of salt at these sites, for only evaporitic dolomite, gypsum, and/or anhydrite were recovered in cores. Among other ionic relationships, influence of evaporites is seen in calcium, magnesium and sulfate concentrations. Record high barium $(216 \mathrm{ppm})$ and strontium (345 ppm) concentrations were observed in sulfate depleted pore fluids of Site 128 in the Hellenic Trench.
\end{abstract}

\section{INTRODUCTION}

The sediments cored on Leg 13 are, for the most part, characterized by high sedimentation rates and usually contain large proportions of terrigenous detritus. Upper Miocene evaporites are widespread throughout the deep basins, and underlie most of the Leg 13 sites. It is to be expected that the influence of both normal diagenesis and underlying evaporites will be seen in the composition of interstitial solutions.

Previous experience has demonstrated that diffusion of salts from evaporite sequences into overlying sediments may strongly influence the composition of interstitial solutions in the superjacent sediments. Increases in interstitial $\mathrm{Na}$ and $\mathrm{Cl}$ concentration with depth have been found to be characteristic of sediments overlying known salt deposits in the Gulf of Mexico (Manheim and Bischoff, 1969; Manheim and Sayles, 1969; Manheim et al., 1972). Data from Leg 10 of the Deep Sea Drilling Project in the Gulf of Mexico and Manheim and Bischoff (1969) demonstrate that large $\mathrm{Mg}$ concentration increases may occur with the more characteristic $\mathrm{Na}$ and $\mathrm{Cl}$ enrichments. Increases in $\mathrm{Ca}$ and $\mathrm{SO}_{4}$ concentration may occur at least locally through the dissolution of gypsum and anhydrite.

Concentration changes resulting from diagenetic reactions not related to evaporites are readily distinguishable from those associated with dissolution and diagenesis of evaporites. Normal marine diagenesis produces little or no significant change in $\mathrm{Na}$ and $\mathrm{Cl}$ concentrations in most cases. In the rare cases where changes are noted, they are depletions. $\mathrm{Mg}$ and $\mathrm{SO}_{4}$ have not been found to be enriched

${ }^{1}$ Site 126 was located below the evaporites in a deep cleft in the Mediterranean Ridge which cuts through the inferred evaporite layer. in normal marine sedimentary sequences in previous DSDP studies, but depletions of both are common. Characteristically, the greatest diagenetic changes in pore fluid composition are observed in rapidly deposited sediments $\left(>5 \mathrm{~cm} / 10^{3}\right.$ years) containing appreciable amounts of terrigenous material.

The analytical methods utilized are those previously employed in this laboratory and described in earlier reports. We gratefully acknowledge the assistance of James O'Neill and Joanne Goudreau in the analysis of these samples.

\section{RESULTS}

The analytical data are presented in Tables 1 and 2 (major and minor constituents, respectively). The data are subject to uncertainties arising chiefly from two sources: $\mathrm{Ca}$ loss due to $\mathrm{CaCO}_{3}$ precipitation, and temperature-ofsqueezing effects. In sediments where $\mathrm{SO}_{4}$ is strongly reduced and alkalinities are increased above $10 \mathrm{meq} / \mathrm{kg}, \mathrm{Ca}$ and alkalinity loss due to $\mathrm{CaCO}_{3}$ precipitation after squeezing has been noted (Gieskes, 1972). Extensive reduction of $\mathrm{SO}_{4}$ is common in the Leg 13 samples, but alkalinities have not exceeded $8 \mathrm{meq} / \mathrm{kg}$. Serious $\mathrm{Ca}$ and alkalinity losses have not been found in studies of such samples (Gieskes, 1972; Sayles et al., 1972). Small losses, however, cannot be ruled out completely. While we believe the depletions of $\mathrm{Ca}$ found are real, the in situ depletions may be somewhat smaller than our data indicate, and the alkalinities reported may be low.

A second source of uncertainty arises from the effects of warming sediments from in situ to laboratory temperatures prior to squeezing. Such treatment leads to depletions of $\mathrm{Ca}$ and $\mathrm{Mg}$, and enrichments of $\mathrm{Na}$ and $\mathrm{K}$ (Mangelsdorf, et al., 1969). Silica is also enriched upon warming (Fanning and Pilson, 1971). These changes do not obscure the existing major ion trends, and with the exception of $\mathrm{K}$, concentra- 
TABLE 1

Major Constituents of Pore Fluids. Values in g/kg $(\% \circ)$ Except as Noted

\begin{tabular}{|c|c|c|c|c|c|c|c|c|c|c|c|c|c|c|c|c|c|c|}
\hline $\begin{array}{cc}\text { Sample } & \\
\text { Desig- } & \text { De } \\
\text { nation } & (\mathrm{m}\end{array}$ & $\begin{array}{l}\text { epth } \\
\text { (m) }\end{array}$ & Age & Description & $\mathrm{Na}^{\mathrm{a}}$ & $\mathrm{Na}^{\mathrm{b}}$ & $\mathrm{K}$ & $\mathrm{Ca}$ & $\mathrm{Mg}$ & $\begin{array}{c}\text { Total } \\
\text { Cations } \\
(\mathrm{meq} / \mathrm{kg})\end{array}$ & $\mathrm{Cl}$ & $\mathrm{SO}_{4}$ & $\underset{(\mathrm{meq} / \mathrm{kg})}{\mathrm{Alk}}$ & $\mathrm{HCO}_{3} \mathrm{c}$ & $\begin{array}{c}\text { Total } \\
\text { Anions } \\
\text { (meq } / \mathrm{kg})\end{array}$ & Sumd & $\begin{array}{l}\text { Refrac- } \\
\text { tometer }\end{array}$ & $\begin{array}{l}\mathrm{H}_{2} \mathrm{O} \\
(\% \circ)\end{array}$ & $\mathrm{pH}$ \\
\hline \multicolumn{19}{|c|}{ Hole $121\left(36^{\circ} 09.65^{\prime} \mathrm{N}, 04^{\circ} 22.43^{\prime} \mathrm{W}\right.$, water depth $1163 \mathrm{~m}$, Alboran Basin) } \\
\hline \multicolumn{4}{|c|}{ Surface ocean water } & 11.2 & 10.9 & 0.40 & 0.44 & 1.31 & 614 & 20.08 & 2.81 & 2.6 & 0.16 & 628 & 36.4 & 36.3 & - & - \\
\hline $121-1-3$ & 65 & Quaternary & $\begin{array}{l}\text { Dark greenish gray marl } \\
\text { ooze, plastic }\end{array}$ & 12.3 & 11.8 & 0.35 & 0.18 & 0.92 & 607 & 21.80 & 0.26 & 8.4 & 0.51 & 628 & 36.3 & 35.8 & 29 & 7.6 \\
\hline $3-3$ & 160 & Quaternary & $\begin{array}{l}\text { Dark greenish gray marl } \\
\text { ooze, plastic }\end{array}$ & 13.4 & 13.1 & 0.31 & 0.48 & 1.02 & 684 & 24.63 & $<0.05$ & 5.8 & 0.35 & 700 & 40.2 & 40.2 & 30 & - \\
\hline $4-4$ & 252 & Quaternary & $\begin{array}{l}\text { Dark greenish gray marl } \\
\text { ooze, plastic }\end{array}$ & 14.9 & 14.7 & 0.26 & 0.76 & 1.19 & 780 & 27.92 & $<0.05$ & 3.2 & 0.20 & 790 & 45.3 & 45.6 & 23 & 7.6 \\
\hline \multicolumn{19}{|c|}{ Hole $122\left(40^{\circ} 26.87^{\prime} \mathrm{N}, 02^{\circ} 37.46^{\prime} \mathrm{E}\right.$, water depth $2146 \mathrm{~m}$, Valencia Trough) } \\
\hline $122-2-2$ & 95 & $\begin{array}{l}\text { Upper } \\
\text { Pliocene }\end{array}$ & $\begin{array}{l}\text { Varicolored brown and } \\
\text { gray alternations of fine } \\
\text { sands and clays }\end{array}$ & 13.0 & 12.2 & 0.34 & 0.83 & 1.31 & 689 & 22.54 & 4.05 & 1.4 & 0.08 & 721 & 42.2 & 41.8 & - & 7.5 \\
\hline \multicolumn{19}{|c|}{ Hole $123\left(40^{\circ} 37.83^{\prime} \mathrm{N}, 02^{\circ} 50.27^{\prime} \mathrm{E}\right.$, water depth $2290 \mathrm{~m}$, Valencia Trough) } \\
\hline $123-1-3$ & 85 & Quaternary & $\begin{array}{l}\text { Olive gray clays and sand, } \\
\text { turbidites }\end{array}$ & 12.5 & 12.0 & 0.35 & 0.41 & 1.34 & 662 & 22.29 & 2.48 & 4.8 & 0.29 & 684 & 39.7 & 40.2 & - & 7.9 \\
\hline $5-2$ & 219 & $\begin{array}{l}\text { Lower } \\
\text { Pliocene }\end{array}$ & $\begin{array}{l}\text { Varicolored gray and } \\
\text { brown nanno to marl } \\
\text { ooze, fine layers of silt } \\
\text { or fine sand }\end{array}$ & 18.0 & 17.5 & 0.31 & 1.30 & 1.30 & 941 & 32.22 & 2.57 & 2.2 & 0.14 & 964 & 55.8 & 56.6 & 24 & 7.4 \\
\hline \multicolumn{19}{|c|}{ Hole $124\left(38^{\circ} 52.38^{\prime} \mathrm{N}, 04^{\circ} 59.69^{\prime} \mathrm{E}\right.$, water depth $2726 \mathrm{~m}$, Balearic Basin) } \\
\hline $124-1$, CC 1 & 128 & Pleistocene & $\begin{array}{l}\text { Varicolored gray terrig- } \\
\text { enous nanno ooze, plas- } \\
\text { tic, mottling }\end{array}$ & 21.2 & 20.5 & 0.33 & 0.69 & 1.44 & 1051 & 37.01 & 1.67 & 2.1 & 0.13 & 1080 & 62.5 & 62.2 & 30 & 7.4 \\
\hline $3, \mathrm{CC} 3$ & 307 & $\begin{array}{l}\text { Lower } \\
\text { Pliocene }\end{array}$ & Light gray nanno ooze & 37.9 & 37.5 & 0.36 & 2.36 & 1.44 & 1875 & 64.92 & 3.02 & 1.0 & 0.6 & 1895 & 110.1 & 110.0 & 27 & 7.2 \\
\hline $4, \mathrm{CC} 3$ & 339 & $\begin{array}{l}\text { Lower } \\
\text { Pliocene }\end{array}$ & $\begin{array}{l}\text { Dark grayish brown } \\
\text { marl ooze }\end{array}$ & 39.2 & 38.6 & 0.35 & 2.48 & 1.36 & 1924 & 66.82 & 3.11 & 0.7 & 0.4 & 1948 & 113.4 & 113.8 & 22 & 7.3 \\
\hline \multicolumn{19}{|c|}{ Holes $125 \& 125 \mathrm{~A}\left(34^{\circ} 37.49^{\prime} \mathrm{N}, 20^{\circ} 25.76^{\prime} \mathrm{E}\right.$, water depth $2782 \mathrm{~m}$, Mediterranean Ridge in the Ionian Basin) } \\
\hline \multicolumn{4}{|c|}{ Surface ocean water } & 12.0 & 11.7 & 0.42 & 0.47 & 1.40 & 660 & 21.52 & 3.10 & 3.3 & 0.20 & 674 & 39.1 & 39.0 & - & - \\
\hline $125-3-5$ & 23 & Quaternary & $\begin{array}{l}\text { Brownish and greenish } \\
\text { gray nanno ooze with } \\
\text { foram and sapropel beds }\end{array}$ & 12.1 & 11.6 & 0.49 & 0.55 & 1.34 & 656 & 21.85 & 2.85 & 2.4 & 0.15 & 671 & 39.3 & 39.0 & 27 & 7.4 \\
\hline $5-1$ & 41 & $\begin{array}{l}\text { Upper } \\
\text { Pliocene }\end{array}$ & $\begin{array}{l}\text { Brown to yellowish brown } \\
\text { nanno ooze, deformed }\end{array}$ & 12.3 & 12.0 & 0.51 & 0.62 & 1.35 & 676 & 22.44 & 2.76 & 2.6 & 0.16 & 692 & 40.1 & 41.2 & 22 & 7.5 \\
\hline $125 \mathrm{~A}-3-\mathrm{CC}$ & 53 & $\begin{array}{l}\text { Upper } \\
\text { Pliocene }\end{array}$ & $\begin{array}{l}\text { Light gray nanno ooze } \\
\text { with forams }\end{array}$ & 12.8 & 12.1 & 0.51 & 0.69 & 1.39 & 688 & 22.93 & 3.33 & 1.9 & 0.11 & 718 & 41.8 & 40.7 & 31 & 7.7 \\
\hline $125-7-4$ & 59 & $\begin{array}{l}\text { Upper } \\
\text { Pliocene }\end{array}$ & $\begin{array}{l}\text { Light yellowish brown } \\
\text { nanno ooze, disturbed by } \\
\text { drilling }\end{array}$ & 12.2 & 12.1 & 0.49 & 0.60 & 1.39 & 683 & 22.25 & 2.97 & 2.6 & 0.16 & 691 & 40.1 & 40.7 & - & 7.7 \\
\hline
\end{tabular}


TABLE 1 - Continued

Holes 125 and 125A (Continued)

\begin{tabular}{|c|c|c|c|c|c|c|c|c|c|c|c|c|c|c|c|c|c|c|}
\hline $125 \mathrm{~A}-6-\mathrm{CC}$ & 80 & $\begin{array}{l}\text { Lower } \\
\text { Pliocene }\end{array}$ & Olive green nanno ooze & 13.2 & 12.5 & 0.57 & 0.98 & 1.47 & 728 & 23.84 & 3.65 & 2.9 & 0.18 & 758 & 43.9 & 44.6 & 29 & 7.9 \\
\hline 9-CC & 102 & $\begin{array}{l}\text { Upper } \\
\text { Miocene }\end{array}$ & $\begin{array}{l}\text { Dark gray dolomite, } \\
\text { plastic }\end{array}$ & 14.8 & 14.7 & 0.70 & 1.49 & 1.91 & 886 & 27.47 & 5.69 & 2.0 & 0.12 & 895 & 52.2 & 52.8 & - & 7.6 \\
\hline \multicolumn{19}{|c|}{ Hole $126\left(35^{\circ} 09.72^{\prime} \mathrm{N}, 21^{\circ}, 25.63^{\prime} \mathrm{E}\right.$, water depth $3730 \mathrm{~m}$, Mediterranean Ridge Cleft in the Ionian Basin) } \\
\hline $126-1-6$ & 41 & Quaternary & $\begin{array}{l}\text { Olive gray marl ooze } \\
\text { homogenous, plastic }\end{array}$ & 12.0 & 11.6 & 0.37 & 0.26 & 1.12 & 619 & 21.71 & 1.15 & - & - & 636 & 36.6 & 36.8 & 28 & 7.7 \\
\hline $2-4$ & 79 & Quaternary & $\begin{array}{l}\text { Gray and brown nanno } \\
\text { ooze and marl ooze }\end{array}$ & 12.3 & 12.1 & 0.33 & 0.36 & 1.08 & 640 & 22.70 & 0.36 & 4.0 & 0.24 & 651 & 37.4 & 37.4 & 32 & 7.5 \\
\hline \multicolumn{19}{|c|}{ Holes $127 \& 127 \mathrm{~A}\left(35^{\circ} 43.90^{\prime} \mathrm{N}, 22^{\circ} 29.81^{\prime} \mathrm{E}\right.$, water depth $4654 \mathrm{~m}$, Northeast Margin, Hellenic Trench) } \\
\hline \multicolumn{4}{|c|}{ Surface ocean water } & 12.1 & 11.9 & 0.42 & 0.48 & 1.42 & 667 & 21.66 & 3.25 & 2.7 & 0.16 & 680 & 39.5 & 39.3 & - & - \\
\hline $127-1-3$ & 22 & Quaternary & $\begin{array}{l}\text { Gray and olive gray nanno } \\
\text { to marl ooze with sand, } \\
\text { disturbed by drilling }\end{array}$ & 12.4 & 11.5 & 0.37 & 0.33 & 1.25 & 630 & 21.59 & 2.64 & 5.3 & 0.32 & 668 & 38.9 & 38.0 & 33 & 7.6 \\
\hline $3-5$ & 42 & Quaternary & $\begin{array}{l}\text { Olive gray marl ooze } \\
\text { disturbed by drilling }\end{array}$ & 12.5 & 12.1 & 0.37 & 0.23 & 1.08 & 635 & 22.27 & 0.87 & 6.1 & 0.37 & 652 & 37.7 & 37.4 & - & 7.7 \\
\hline $127 \mathrm{~A}-3-4$ & 50 & Quaternary & $\begin{array}{l}\text { Dark gray to olive gray } \\
\text { marl ooze, homogeneous }\end{array}$ & 13.3 & 12.8 & 0.36 & 0.20 & 1.04 & 663 & 24.00 & $<0.05$ & 7.5 & 0.46 & 684 & 39.4 & 39.0 & 33 & 7.7 \\
\hline $4-4$ & 77 & Quaternary & $\begin{array}{l}\text { Gray and dark gray marl } \\
\text { ooze, plastic, gassy }\end{array}$ & 16.6 & 16.2 & 0.42 & 0.35 & 1.20 & 832 & 30.00 & $<0.05$ & 2.0 & 0.12 & 848 & 48.7 & 48.4 & - & 7.7 \\
\hline $127-4-2$ & 91 & Quaternary & $\begin{array}{l}\text { Dark greenish gray marl } \\
\text { ooze, graded sand and silt }\end{array}$ & 16.0 & 15.6 & 0.40 & 0.35 & 1.21 & 804 & 29.22 & $<0.05$ & 1.9 & 0.12 & 825 & 47.3 & 47.8 & 29 & 7.6 \\
\hline $6-5$ & 108 & Quaternary & $\begin{array}{l}\text { Dark greenish gray marl } \\
\text { ooze, plastic }\end{array}$ & 19.7 & 19.5 & 0.45 & 0.55 & 1.52 & 1012 & 36.08 & $<0.05$ & 3.5 & 0.21 & 1021 & 58.5 & 58.8 & - & 7.2 \\
\hline $8-4$ & 172 & Quaternary & $\begin{array}{l}\text { Olive gray nanno ooze with } \\
\text { olive black sand layer } \\
\text { below }\end{array}$ & 37.9 & 34.4 & 0.55 & 1.00 & 1.33 & 1670 & 64.41 & 0.18 & 1.1 & 0.06 & 1821 & 105.4 & 104.5 & 27 & 7.3 \\
\hline $9, \mathrm{CC}$ & 233 & Quaternary & $\begin{array}{l}\text { Varicolored gray silt to } \\
\text { clay, nannos abundant }\end{array}$ & 42.5 & 42.1 & 0.71 & 3.29 & 2.26 & 2199 & 76.81 & 2.40 & 0.7 & 0.04 & 2217 & 128.0 & 130.9 & - & 7.2 \\
\hline $10, \mathrm{CC}$ & 284 & Quaternary & Olive gray nanno ooze & 44.1 & 44.9 & 0.80 & 3.62 & 2.33 & 2345 & 82.08 & 3.09 & 1.2 & 0.07 & 2380 & 136.1 & 140.8 & 24 & 7.0 \\
\hline $11, \mathrm{CC}$ & 308 & Quaternary & Light gray nanno ooze & 43.7 & 41.2 & 0.73 & 3.17 & 2.27 & 2156 & 78.25 & 2.82 & 0.8 & 0.05 & 2266 & 131.0 & 132.6 & 27 & 7.2 \\
\hline $12, \mathrm{CC}$ & 336 & Quaternary & $\begin{array}{l}\text { Olive gray nanno ooze, } \\
\text { plastic to stiff }\end{array}$ & 46.7 & 45.9 & 0.80 & 2.87 & 2.45 & 2362 & 82.91 & 2.79 & 0.7 & 0.04 & 2397 & 138.6 & 140.8 & 23 & 7.3 \\
\hline $15, \mathrm{CC}$ & 427 & Quaternary & $\begin{array}{l}\text { Olive gray nanno ooze, } \\
\text { plastic to stiff }\end{array}$ & 52.4 & 50.9 & 0.79 & 2.17 & 2.01 & 2510 & 89.42 & 2.51 & 1.1 & 0.07 & 2575 & 149.4 & 150.7 & - & 7.5 \\
\hline \multicolumn{19}{|c|}{ Hole $128\left(35^{\circ} 42.58^{\prime} \mathrm{N}, 22^{\circ} 28.10^{\prime} \mathrm{E}\right.$, water depth $4640 \mathrm{~m}, \mathrm{SW}$ margin Hellenic Trench) } \\
\hline $128-2-4$ & 56 & Quaternary & Olive gray marl ooze, gassy & 15.5 & 15.2 & 0.45 & 0.32 & 1.41 & 805 & 28.72 & $<0.05$ & 4.8 & 0.29 & 820 & 46.9 & 48.4 & 28 & 7.6 \\
\hline $5-6$ & 153 & Quaternary & $\begin{array}{l}\text { Olive gray nanno ooze } \\
\text { with graded layers of } \\
\text { sand, gassy }\end{array}$ & 26.0 & 25.3 & 0.55 & 1.65 & 3.68 & 1499 & 54.32 & $<0.05$ & 1.1 & 0.06 & 1533 & 86.3 & 90.8 & 24 & 7.6 \\
\hline $7-6$ & 252 & Quaternary & $\begin{array}{l}\text { Olive gray nanno ooze, } \\
\text { plastic to stiff, gassy }\end{array}$ & 38.1 & 36.8 & 0.73 & 1.55 & 3.68 & 1999 & 72.98 & $<0.05$ & 0.6 & 0.04 & 2059 & 117.1 & 121.6 & 23 & 7.8 \\
\hline $8, \mathrm{CC}$ & 313 & Quaternary & $\begin{array}{l}\text { Olive gray and dark } \\
\text { greenish gray marl ooze }\end{array}$ & 43.2 & 42.3 & 0.59 & 1.36 & 3.17 & 2186 & 78.79 & $<0.05$ & 0.9 & 0.06 & 2223 & 127.2 & 132.0 & 23 & 7.3 \\
\hline
\end{tabular}


TABLE 1 - Continued

\begin{tabular}{|c|c|c|c|c|c|c|c|c|c|c|c|c|c|c|c|c|c|c|}
\hline $\begin{array}{l}\text { Sample } \\
\text { Desig- } \\
\text { nation }\end{array}$ & $\begin{array}{l}\text { Depth } \\
\text { (m) }\end{array}$ & Age & Description & $\mathrm{Na}^{\mathrm{a}}$ & $\mathrm{Na}^{\mathrm{b}}$ & $\mathrm{K}$ & $\mathrm{Ca}$ & $\mathrm{Mg}$ & $\begin{array}{c}\text { Total } \\
\text { Cations } \\
\text { (meq } / \mathrm{kg})\end{array}$ & $\mathrm{Cl}$ & $\mathrm{SO}_{4}$ & $\begin{array}{c}\text { Alk. } \\
\text { (meq } / \mathrm{kg})\end{array}$ & $\mathrm{HCO}_{3}{ }^{\mathrm{c}}$ & $\begin{array}{c}\text { Total } \\
\text { Anions } \\
\text { (meq } / \mathrm{kg})\end{array}$ & Sum $^{\mathrm{d}}$ & $\begin{array}{l}\text { Refrac- } \\
\text { tometer }\end{array}$ & $\begin{array}{l}\mathrm{H}_{2} \mathrm{O}^{\mathrm{e}} \\
(\% \circ)\end{array}$ & $\mathrm{pH}$ \\
\hline \multicolumn{19}{|c|}{ Hole $130\left(33^{\circ} 36.31^{\prime} \mathrm{N}, 27^{\circ} 51.99^{\prime} \mathrm{E}\right.$, water depth 2979 , Mediterranean Ridge in the Levantine Basin) } \\
\hline $130-1-3$ & 31 & Quaternary & $\begin{array}{l}\text { Light gray to olive gray } \\
\text { nanno ooze, odor of } \mathrm{H}_{2} \mathrm{~S}\end{array}$ & 11.8 & 11.3 & 0.41 & 0.32 & 0.93 & 595 & 21.18 & 0.51 & 7.1 & 0.43 & 614 & 35.6 & 35.8 & 35 & 7.8 \\
\hline $6, \mathrm{CC}$ & 418 & Quaternary & $\begin{array}{l}\text { Dark gray clay and green- } \\
\text { ish gray nanno ooze, sharp } \\
\text { contact between Nile clay } \\
\text { and pelagic deposits }\end{array}$ & 13.2 & 13.1 & 0.31 & 0.50 & 1.00 & 683 & 24.41 & 0.08 & 0.6 & 0.04 & 690 & 39.5 & 40.2 & 31 & 7.9 \\
\hline \multicolumn{19}{|c|}{ Hole $132\left(40^{\circ} 15.70^{\prime} \mathrm{N}, 11^{\circ} 26.47^{\prime} \mathrm{E}\right.$, water depth $2835 \mathrm{~m}$, Tyrrhenian Basin) } \\
\hline $132-2-3$ & 16 & Quaternary & $\begin{array}{l}\text { Light olive gray nanno } \\
\text { ooze }\end{array}$ & 11.7 & 11.6 & 0.43 & 0.54 & 1.37 & 654 & 21.29 & 2.76 & 2.7 & 0.16 & 660 & 38.3 & 39.0 & 47 & 7.4 \\
\hline $4-5$ & 33 & Quaternary & Yellow brown nanno ooze & 11.6 & 11.4 & 0.39 & 0.60 & 1.40 & 651 & 21.36 & 2.56 & 3.0 & 0.18 & 658 & 38.1 & 38.5 & 40 & 7.3 \\
\hline $6-3$ & 48 & Quaternary & $\begin{array}{l}\text { Light olive gray nanno } \\
\text { ooze, moderately } \\
\text { deformed }\end{array}$ & 11.7 & 11.5 & 0.35 & 0.68 & 1.42 & 660 & 21.42 & 3.03 & 3.1 & 0.19 & 670 & 38.8 & 39.6 & 33 & 7.2 \\
\hline $8-4$ & 68 & Quaternary & $\begin{array}{l}\text { Olive gray nanno ooze, } \\
\text { deformed }\end{array}$ & 11.9 & 11.6 & 0.35 & 0.79 & 1.44 & 670 & 21.81 & 3.31 & 2.3 & 0.14 & 686 & 39.7 & 40.2 & 35 & 7.2 \\
\hline $10-5$ & 87 & $\begin{array}{l}\text { Upper } \\
\text { Pliocene }\end{array}$ & Brown foram-nanno ooze & - & 11.7 & 0.35 & $(1.24)^{\mathrm{f}}$ & 1.48 & - & 22.16 & 3.52 & 2.0 & 0.12 & 700 & 40.4 & 41.8 & 31 & 7.2 \\
\hline $12-5$ & 105 & $\begin{array}{l}\text { Upper } \\
\text { Pliocene }\end{array}$ & $\begin{array}{l}\text { Light olive gray foram- } \\
\text { nanno ooze }\end{array}$ & 12.0 & 12.0 & 0.34 & 1.10 & 1.52 & 712 & 22.44 & 3.79 & 2.1 & 0.13 & 713 & 41.3 & 42.4 & 32 & 7.2 \\
\hline $15-4$ & 130 & $\begin{array}{l}\text { Lower } \\
\text { Pliocene }\end{array}$ & $\begin{array}{l}\text { Pale yellow foram-nanno } \\
\text { ooze }\end{array}$ & 12.8 & 12.5 & 0.34 & 1.37 & 1.58 & 750 & 23.86 & 4.21 & 1.7 & 0.11 & 762 & 44.3 & 45.6 & 29 & 7.4 \\
\hline $17-4$ & 148 & $\begin{array}{l}\text { Lower } \\
\text { Pliocene }\end{array}$ & $\begin{array}{l}\text { Light olive gray nanno } \\
\text { ooze }\end{array}$ & 12.1 & 13.5 & 0.37 & 1.64 & 1.75 & 820 & 23.62 & 4.53 & 1.0 & 0.06 & 761 & 44.1 & 45.6 & 29 & 7.0 \\
\hline $19-3$ & 168 & $\begin{array}{l}\text { Lower } \\
\text { Pliocene }\end{array}$ & $\begin{array}{l}\text { Light olive gray nanno } \\
\text { ooze }\end{array}$ & 12.4 & 12.3 & 0.32 & 1.60 & 1.64 & 758 & 23.64 & 4.65 & 1.1 & 0.07 & 764 & 44.3 & 46.2 & 29 & 6.8 \\
\hline
\end{tabular}

${ }^{\mathrm{a}}$ Sodium determined by difference between anions and cations excluding Na.

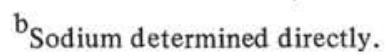

${ }^{\mathrm{c}} \mathrm{HCO}_{3}$ is calculated from total alkalinity, assuming this is entirely due to bicarbonate ion.

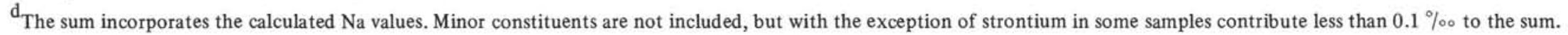

$\mathrm{e}_{\mathrm{pH}}$ and water content are taken from shipboard summaries.

$\mathrm{f}_{\text {Value determined by difference. }}$ 
31.1. INTERSTITIAL WATER STUDIES ON SMALL CORE SAMPLES

TABLE 2

Minor Constituents of Pore Fluids. Concentrations in $\mathrm{mg} / \mathrm{kg}$ (ppm)

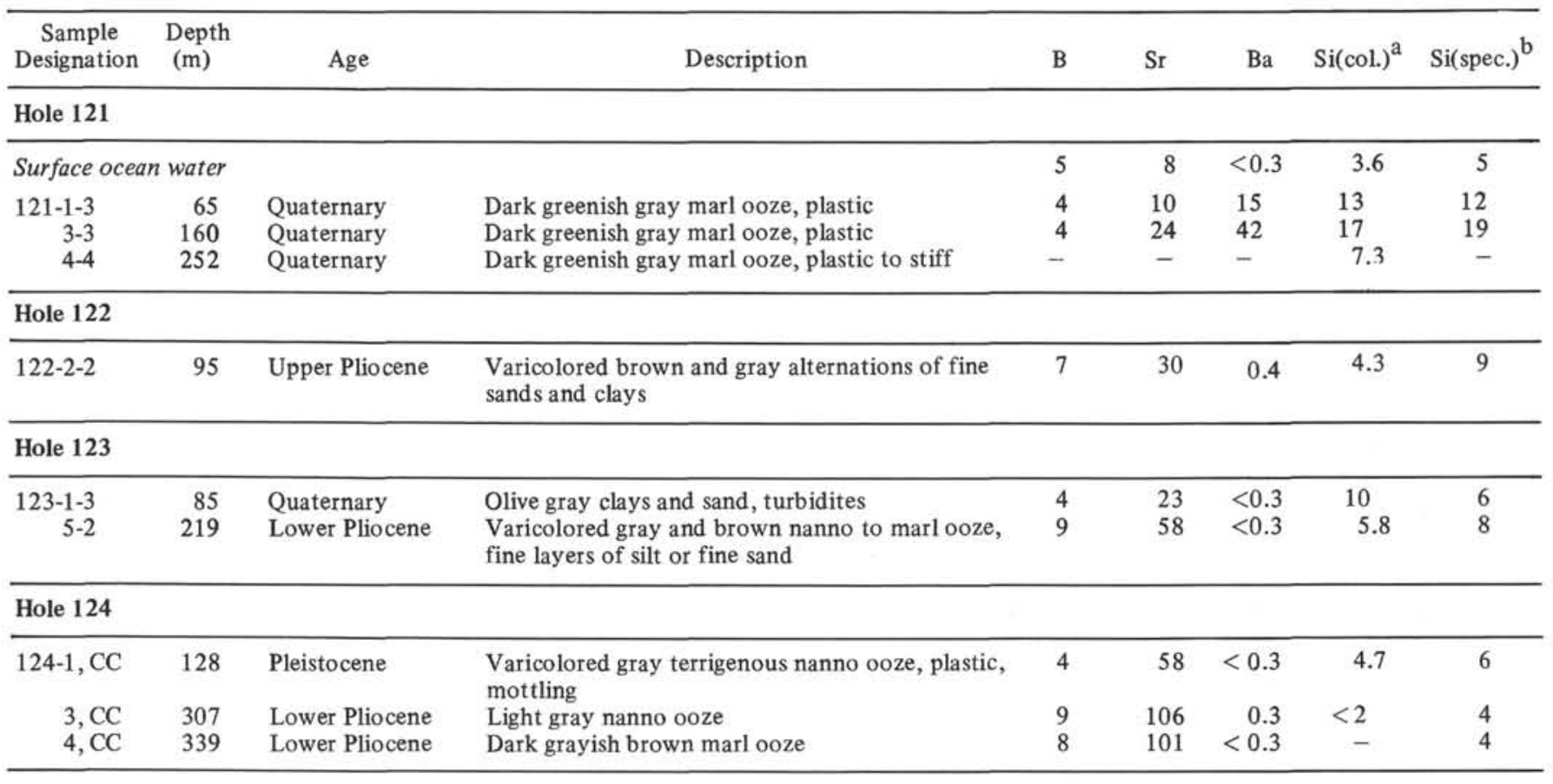

\section{Holes 125 and 125A}

\begin{tabular}{|c|c|c|c|c|c|c|c|c|}
\hline \multicolumn{4}{|c|}{ Surface ocean water } & 5 & 9 & $<0.3$ & $<2$ & $<2$ \\
\hline $125-3-5$ & 23 & Quaternary & $\begin{array}{l}\text { Brownish and greenish gray nanno ooze with } \\
\text { foram and sapropel beds }\end{array}$ & 5 & 17 & $<0.3$ & 4.4 & 6 \\
\hline $5-1$ & 41 & Upper Pliocene & Brown to yellowish brown nanno ooze, deformed & 8 & 19 & $<0.3$ & 3.0 & 5 \\
\hline $125-7-4$ & 59 & Upper Pliocene & $\begin{array}{l}\text { Light yellowish brown nanno ooze, disturbed by } \\
\text { drilling }\end{array}$ & 9 & 19 & $<0.3$ & 9.8 & 10 \\
\hline $125 \mathrm{~A}-6, \mathrm{CC}$ & 80 & Early Pliocene & Olive green nanno ooze & 6 & 30 & $<0.3$ & 4.8 & 5 \\
\hline
\end{tabular}

Hole 126

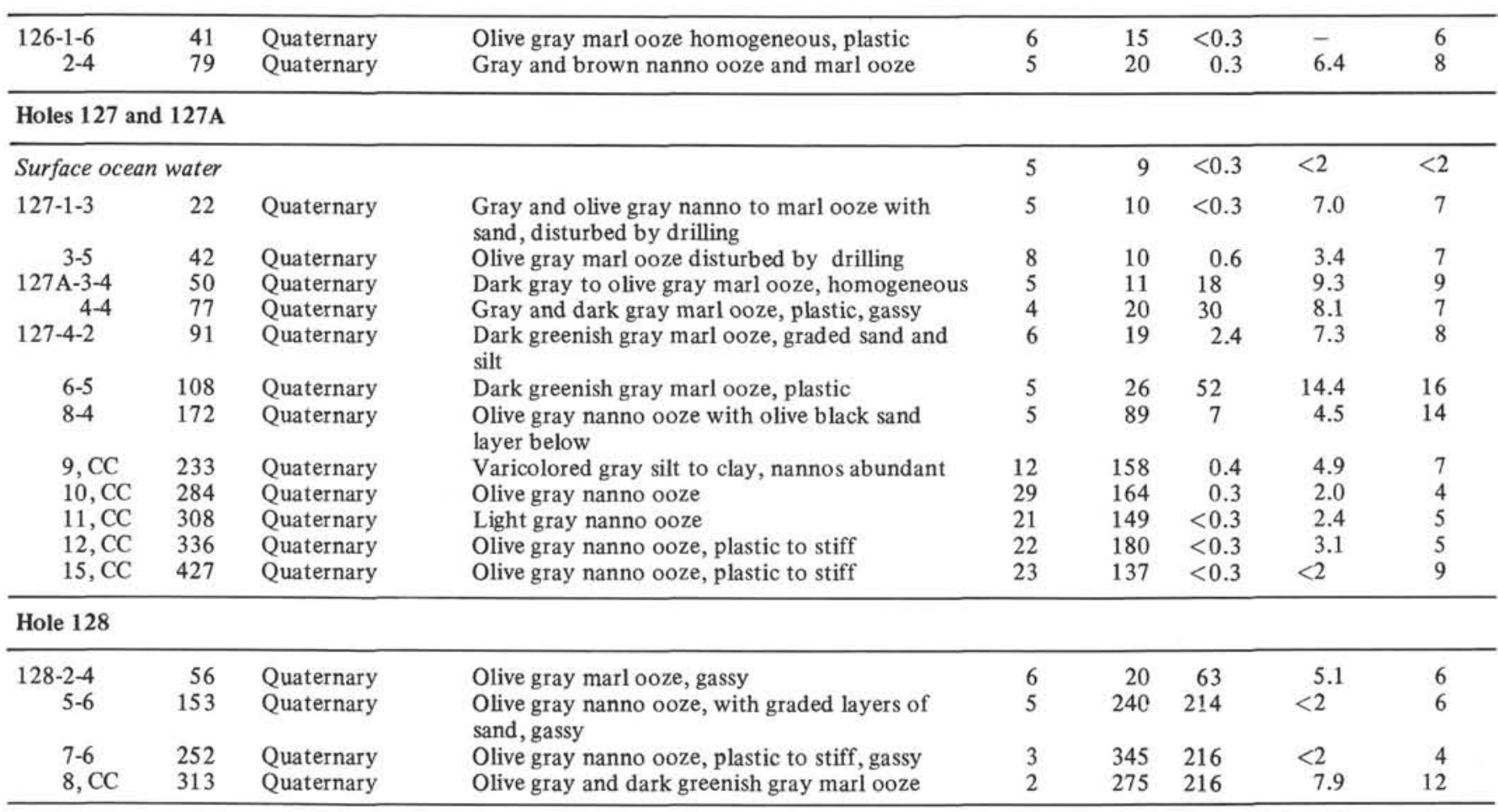


TABLE 2 - Continued

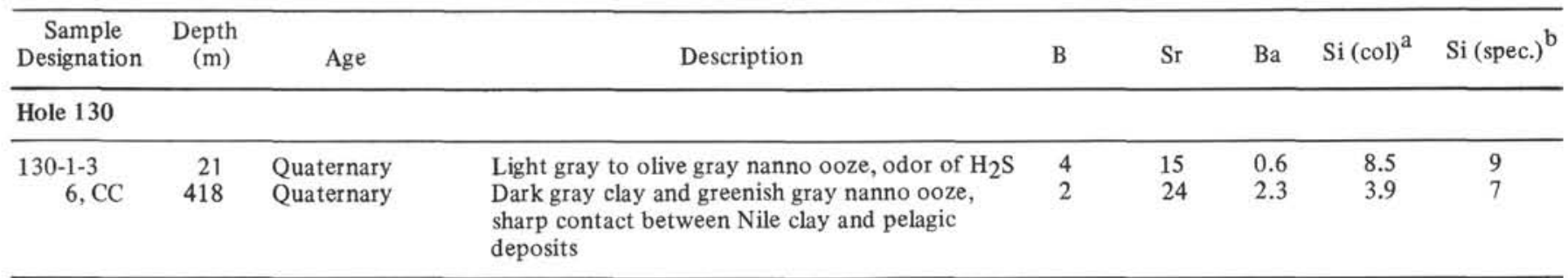

Hole 132

\begin{tabular}{|c|c|c|c|c|c|c|c|c|}
\hline $132-2-3$ & 16 & Quaternary & Light olive gray nanno ooze & 7 & 16 & $<0.3$ & 10. & 9 \\
\hline 4-5 & 33 & Quaternary & Yellow brown nanno ooze & 8 & 22 & $<0.3$ & 4.3 & 5 \\
\hline $6-3$ & 48 & Quaternary & $\begin{array}{l}\text { Light olive gray nanno ooze, moderately } \\
\text { deformed }\end{array}$ & 7 & 23 & $<0.3$ & 13 & 10 \\
\hline $8-4$ & 68 & Quaternary & Olive gray nanno ooze, deformed & 6 & 30 & $<0.3$ & 7.2 & 8 \\
\hline $10-5$ & 87 & Upper Pliocene & Brown foram-nanno ooze & 7 & 35 & $<0.3$ & 4.5 & 7 \\
\hline $12-5$ & 105 & Upper Pliocene & Light olive gray foram-nanno ooze & 8 & 37 & $<0.3$ & 5.3 & 7 \\
\hline $15-4$ & 130 & Lower Pliocene & Pale yellow foram-nanno ooze & 8 & 41 & $<0.3$ & 4.7 & 6 \\
\hline $17-4$ & 148 & Lower Pliocene & Light olive gray nanno ooze & 7 & 41 & $<0.3$ & $<2$ & 6 \\
\hline $19-4$ & 168 & Lower Pliocene & Light olive gray nanno ooze & 8 & 47 & $<0.3$ & 5.7 & 6 \\
\hline
\end{tabular}

tions are altered 5 per cent or less (Sayles et al., 1972). K concentrations may be significantly affected. Gains of up to $.08 \mathrm{~g} / \mathrm{kg}$ have been observed (Bischoff et al., 1970; Sayles et al., 1972).

Enrichments of $\mathrm{Na}$ and $\mathrm{Cl}$ are found at all of the sites sampled for interstitial water studies. At Sites 122, Valencia Trough, and 126, Mediterranean Ridge, the $\mathrm{Cl}$ increases are small but above analytical uncertainty. Evaporation prior to squeezing or inadequate sealing of storage vessels could have produced these small increases, but the increase in $\mathrm{Cl}$ with depth at Site 126 suggests the conclusion that real $\mathrm{Cl}$ gradients exist at least at this site. The increases in $\mathrm{Na}$ and $\mathrm{Cl}$ found at all of the other sites sampled on Leg 13 are large enough to avoid uncertainty as to the existence of in situ enrichments. Where sufficient samples exist, these increases can be seen to be roughly linear as a function of depth. $\mathrm{Cl}$ concentrations of 25 to $30 \mathrm{~g} / \mathrm{kg}$ are common with maximum values of 89 and $79 \mathrm{~g} / \mathrm{kg}$ occurring at Sites 127 and 128 , respectively.

At Sites 122 and 124 in the western Mediterranean, 125 and 127 in the eastern Mediterranean, and 132 in the Tyrrhenian Sea, the influence of underlying evaporites appears to exert a dominant influence on $\mathrm{SO}_{4}$ concentrations and in some instances on $\mathrm{Mg}(124,127,132)$. Ca concentrations appear to reflect the interaction of both diagenetic and evaporitic influences. Gypsum or anhydrite was recovered in cores from each of these sites except 127 where penetration was terminated before reaching the level of the evaporite formation. Not surprisingly, the pore waters commonly are characterized by $\mathrm{Ca}$ and $\mathrm{SO}_{4}$ enrichments. Relative to standard seawater $\left(\mathrm{Ca}=0.41 \mathrm{~g} / \mathrm{kg}, \mathrm{SO}_{4}\right.$ $=2.71 \mathrm{~g} / \mathrm{kg}$ ), nine-fold $\mathrm{Ca}$ increases (to $3.62 \mathrm{~g} / \mathrm{kg}$ ) and two-fold $\mathrm{SO}_{4}$ enrichments (to $5.69 \mathrm{~g} / \mathrm{kg}$ ) are found. $\mathrm{Mg}$ concentrations in excess of seawater $(1.29 \mathrm{~g} / \mathrm{kg})$ are observed at Sites 124 (slight), 125, 127 and 132, reaching concentrations of $2.45 \mathrm{~g} / \mathrm{kg}$ at Site 127 with values exceeding $1.5 \mathrm{~g} / \mathrm{kg}$ common.
At Sites 121 (Alboran Basin), 123 (Valencia Trough), and 126 and 130 on the Mediterranean Ridge, $\mathrm{Ca}, \mathrm{Mg}$ and $\mathrm{SO}_{4}$ concentrations chiefly reflect the influence of diagenetic reactions rather than that of evaporites at depth. In the absence of evaporites, $\mathrm{Mg}$ concentrations have been found to invariably decrease with depth. Depletions of $\mathrm{Mg}$ may be related to dolomitization (Sayles et al., 1972; Broecker, 1972) or to silicate reactions such as those proposed by Drever (1971). Mg depletions are found in Quaternary sediments at Sites 121,126,130 and the upper portion of 127. The depletions of $\mathrm{Mg}$ (to $0.9-1.1 \mathrm{~g} / \mathrm{kg}$ ) are compatible with earlier DSDP pore water data. Depletions of $\mathrm{SO}_{4}$ are also a common product of diagenesis and result from bacterial reduction of $\mathrm{SO}_{4}$. Depletion of more than 80 per cent of the original $\mathrm{SO}_{4}$ present is found at Sites 121,126 and 130. Smaller losses are found at Site 123. Ca depletion commonly accompanies strong $\mathrm{SO}_{4}$ reduction at least in the upper portions of such sites (Gieskes, 1972; Sayles, et al., 1972). Losses of $\mathrm{Ca}$ are found at Sites 121, 126 and 130 , those sites marked by $\mathrm{SO}_{4}$ depletion. With the possible exception of Sample 130-1-3, we believe the Ca losses are too large to be explained as an artifact resulting from the precipitation of $\mathrm{CaCO}_{3}$ after squeezing. Once $\mathrm{SO}_{4}$ reduction is virtually complete, further $\mathrm{Ca}$ depletion does not occur. At depth in these sites, depletion actually gives way to gradual $\mathrm{Ca}$ enrichment. This effect can be seen in the lowermost samples of Sites 121, 126 and 130. At sites where no appreciable $\mathrm{SO}_{4}$ reduction occurs, $\mathrm{Ca}$ enrichment commonly is seen over the entire hole (cf. data Legs 8 and 15). Thus, all of the $\mathrm{Ca}, \mathrm{Mg}$ and $\mathrm{SO}_{4}$ changes occurring at Sites $121,123,126$ and 130 can be adequately explained within the framework of diagenetic alterations noted in previous interstitial water studies.

Site 128 in the Hellenic Trench exhibits behavior characteristic of both evaporite influence and diagenesis. Concentrations of $\mathrm{SO}_{4}$ are below our detection limit (.05 $\mathrm{g} / \mathrm{kg}$ ) in all samples from this site, reflecting complete 
utilization in diagenetic reduction. The observed Ca enrichments (below $56 \mathrm{~m}$ ) may reflect either diagenetic or evaporitic influence. $\mathrm{Mg}$ is strongly enriched reaching concentrations of $3.26 \mathrm{~g} / \mathrm{kg}$, the highest value recorded on Leg 13. As noted above, such enrichment has only been found where evidence of underlying evaporites exists. Site 127 , located a few kilometers from Site 128, also exhibits a somewhat split personality. In the upper portions of this site $(<177 \mathrm{~m})$ diagenetic reactions hold sway, but below this the influence of evaporites on $\mathrm{Mg}$ and $\mathrm{SO}_{4}$ can be seen.

The concentrations of minor elements in the samples from Leg 13 exhibit some of the largest deviations from seawater found during the Deep Sea Drilling Project. Enrichments of $\mathrm{Sr}$ reached $180 \mathrm{ppm}$ and $345 \mathrm{ppm}$ in samples from Sites 127 and 128, respectively. Modest enrichments of 20 to $60 \mathrm{ppm}$ are found in all of the other sites for which samples are available. Such modest enrichments are quite common in interstitial solutions of calcareous sediments from the Pacific Ocean and have been discussed in earlier leg reports (e.g. Legs 7 and 8). Ba concentrations markedly higher than any reported previously by us are found at Site 128 (up to $216 \mathrm{ppm}$ ), and unusually high values are found at Sites 121 and 127. As has been noted previously, Ba enrichments are characteristic of sites exhibiting extensive $\mathrm{SO}_{4}$ reduction, indicating that barite solubility is the primary control of concentration. The extremely high values at Site 128 may well represent other unidentified influences, however. The reduction of $\mathrm{SO}_{4}$ to our detection limit is common, but concentrations even approaching those at 128 have not been seen previously. The unusually high $\mathrm{Mg}$ and $\mathrm{Sr}$ also suggest abnormal conditions at this site. While the $\mathrm{B}$ data exhibit considerable scatter, real enrichment relative to seawater is usually found at the Leg 13 sites where evaporites appear to influence $\mathrm{Mg}$ or $\mathrm{SO}_{4}$. Concentrations commonly fall in the range 6 to $9 \mathrm{ppm}$ at these sites. At $127, \mathrm{~B}$ is strongly enriched to 20 to $29 \mathrm{ppm}$ in the lower portion of the hole.

\section{DISCUSSION}

The existence of $\mathrm{Na}$ and $\mathrm{Cl}$ gradients at all but two sites for which interstitial water sampling was carried out indicate that halite or brine-containing evaporites exist at depth at all of these sites. The two remaining sites (122 and 126) exhibit small chloride enrichments, but sampling was too sparse and shallow to definitely prove the existence of $\mathrm{Na}$ and $\mathrm{Cl}$ enrichments and gradients characteristic of sediments overlying salt deposits. Although halite was not recovered at any of the above sites, we believe that $\mathrm{Na}$ and $\mathrm{Cl}$ data provide sufficient evidence for concluding that such sediments do exist at depth at all of the sites except perhaps 122 and $126 .^{1}$ This conclusion is in agreement with seismic data which also suggest that a halite layer exists below the deepest penetration at these sites.

Site 121 was terminated in a schist which, in the preliminary report, is presumed to be basement. The interstitial waters of Site 121 are characterized by $\mathrm{Na}$ and $\mathrm{Cl}$ gradients typical of sediments overlying salt. This site was drilled over a basement high onto which Pliocene and Miocene sediments lap unconformably (W. B. F. Ryan, personal communication). Thus, some lateral movement of brines from the Miocene or Pliocene sediments must occur to account for the observed gradients. There is little question that halite or brine-containing sediments do exist near this site.

Enrichments of $\mathrm{SO}_{4}$ have not been found previously in interstitial waters sampled by the DSDP. There is little doubt that the enhanced $\mathrm{SO}_{4}$ concentrations, common in these pore fluids, result from the dissolution of gypsum or anhydrite. With one exception, the $\mathrm{SO}_{4}$ enrichments are found at sites where evaporitic gypsum or anhydrite was recovered (Sites 122, 124, 125, 132). Site 132 appears to provide the most straightforward example of the influence of anhydrite dissolution upon overlying sediments. As seen in Figure 1, $\Delta \mathrm{Ca}$ and $\Delta \mathrm{SO}_{4}(\Delta=$ change in pore water concentration relative to seawater) vary linearly suggesting that dissolution and diffusion are responsible for the observed concentration increases. The slope of the $\Delta \mathrm{Ca}-\Delta \mathrm{SO}_{4}$ correspondence is not 1 as required by simple dissolution, but rather 1.5. Consequently, some concurrent reaction must either supply $\mathrm{Ca}$ or remove a fraction of the $\mathrm{SO}_{4}$ released by dissolution. Both reactions have been frequently observed in previous DSDP studies. At the other sites where evaporitic sediments were cored, no simple and consistent relationship between $\mathrm{Ca}$ and $\mathrm{SO}_{4}$ can be determined. Insufficient data are available at Sites 122 and 124, and the data of 125 exhibit no consistent trend.

In several earlier legs of the DSDP (e.g., 7, 8, and 15) a nearly linear correspondence between $\mathrm{Ca}$ and $\mathrm{Sr}$ enrichment was found to exist. A similar correspondence appears to hold for all of the Leg 13 sites where sufficient data are available for interpretation. At least at Site 132 the linear variations of $\Delta \mathrm{Ca}, \Delta \mathrm{SO}_{4}$ and $\Delta \mathrm{Sr}$ indicate that the $\mathrm{Sr}$ is

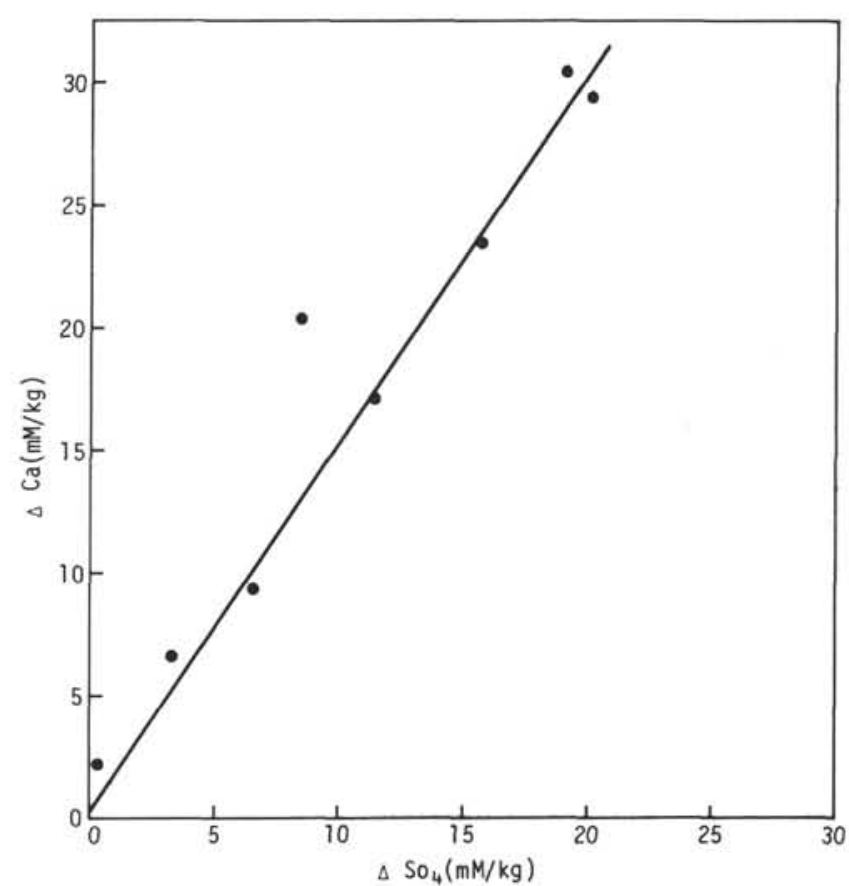

Figure 1. The variation in $\mathrm{Ca}$ enrichment with $\mathrm{SO}_{4}$ enrichment $(\Delta=$ pore water concentration - standard seawater concentration.) Data are for Site 132 only. 
released during dissolution and possibly recrystallization of gypsum and anhydrite. The slopes of the data plots in Figure 2 are in the range 60 to 70 . This requires that between 1 and 2 per cent of the $\mathrm{Ca}$ ion sites in a presumed $\mathrm{CaSO}_{4}$ phase be occupied by $\mathrm{Sr}$ if solution of $\mathrm{CaSO}_{4}$ is responsible for the observed enrichments. Since most marine gypsum and anhydrite rocks contain $<0.2$ per cent Sr (Stewart, 1963; F. T. Manheim, unpublished data), we presume that much of the $\mathrm{Sr}$ is released during recrystallization.

The abnormally high concentrations of $\mathrm{Mg}$ observed at Sites 127, 128 and 132 are difficult to explain. Normal diagenetic changes lead to the depletion of $\mathrm{Mg}$, whereas the increases noted above are believed due to the influence of evaporitic sediments. Late stage evaporites, in particular, contain readily soluble Mg-bearing minerals and could supply $\mathrm{Mg}$. The data argue against any simple explanation, however, for at least Sites 127 and $128, \mathrm{Mg}$ concentrations pass through a maximum. This is also true of $\mathrm{Mg}$ at 132 , but the drop in the lowermost sample is small and a maximum is not well established. Maxima in $\mathrm{Mg} v s$. depth

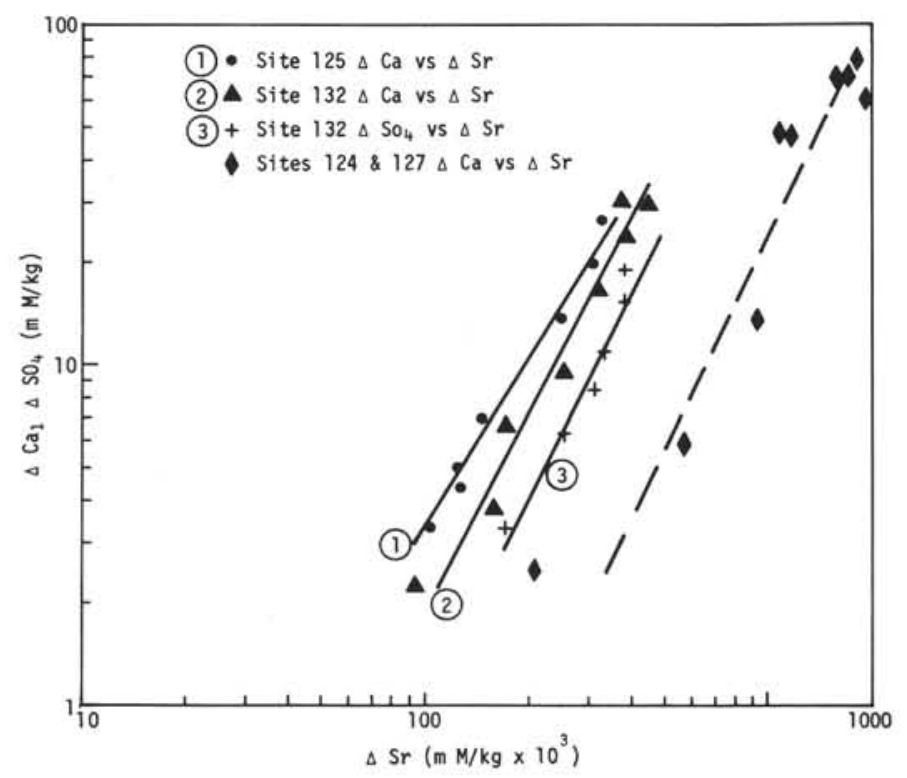

Figure 2. The variation of $\mathrm{Sr}$ enrichment with $\mathrm{Ca}$ and $\mathrm{SO}_{4}$ enrichment for Site 132 and Ca enrichment only for Sites 124,125 and 127. $\Delta=$ pore water concentration - standard seawater concentration). profiles have also been found associated with known or inferred evaporites in the Gulf of Mexico by Manheim and Bischoff (1969) in core 26 and at DSDP Site 92 (Leg 10). Such maxima cannot be explained by diffusive fluxes of $\mathrm{Mg}$ from late stage evaporites at depth, and diagenetic reactions must be responsible. This type of $\mathrm{Mg} v s$. depth distribution is associated with evaporitic sediments, but we do not know the nature of the reactions occurring.

\section{REFERENCES}

Bischoff, J. L., Greer, R. E. and Luistros, A. O., 1970. Composition of interstitial waters of marine sediments: temperature of squeezing effect. Science. 167, 1245.

Broecker, W. S., 1972. Initial Reports of the Deep Sea Drilling Project, Volume 15 (in press).

Drever, J. I., 1971. Magnesium-iron replacement in clay minerals in anoxic marine sediments. Science. 172, 1334.

Fanning, D. K. and Pilson, M. E. Q., 1971. Interstitial silica and $\mathrm{pH}$ in marine sediments: some effects of sampling procedure. Science. 173, 1228.

Gieskes, J. M. Interstitial water studies, Leg 15. In Edgar, N. T. and Saunders, J. B., et al., 1972. Initial Reports of the Deep Sea Drilling Project, Volume 15. Washington (U.S. Government Printing Office) (in preparation).

Mangelsdorf, P. C., Jr., Wilson, T. R. S. and Daniell, E., 1969. Potassium enrichments in interstitial waters of marine sediments. Science. 165, 171.

Manheim, F. T. and Bischoff, J. L., 1969. Geochemistry of pore waters from Shell Oil Company drill holes on the continental slope of the northern Gulf of Mexico. Chem. Geol. 4, 63.

Manheim, F. T. and Sayles, F. L., 1969. Interstitial Water studies on small core samples, Deep Sea Drilling Project, Leg 1. In Ewing, M. and Worzel, J. L., et al., 1969. Initial Reports of the Deep Sea Drilling Project, Volume 1. Washington (U.S. Government Printing Office), 403.

Manheim, F. T., Sayles, F. L. and Waterman, L. S. Interstitial water studies on small core samples, Leg 10. In Worzel, J. L. and Bryant, W. R., et al., Initial Reports of the Deep Sea Drilling Project, Volume 10. Washington, D. C. (U.S. Government Printing Office) (in preparation).

Sayles, F. L., Manheim, F. T. and Waterman, L. S. Interstitial water studies on small core samples, Leg 15. In Edgar, N. T. and Saunders, J. B., et al., Initial Reports of the Deep Sea Drilling Project, Volume 15. Washington, D.C. (U.S. Government Printing Office) (in preparation).

Stewart, F. H., 1963. Marine Evaporites. U.S. Geol. Surv. Prof. Paper, 440-y. 